\title{
Disaster Information Collecting/Providing Service for Local Residents
}

\author{
Yuichi Takahashi $^{1}$, Daiji Kobayashi ${ }^{2}$, and Sakae Yamamoto ${ }^{1}$ \\ ${ }^{1}$ Department of Management Science, Tokyo University of Science, 1-3 Kagurazaka Shinjuku \\ Tokyo Japan \\ \{yt, sakae\}@hci.ms.kagu.tus.ac.jp \\ ${ }^{2}$ Faculty of Photonics Science Department of Global System Design, Chitose Institute of \\ Science and Technology, 758-65 Bibi Chitose Hokkaido Japan \\ d-kobaya@photon.chitose.ac.jp
}

\begin{abstract}
It has been pointed out that when people lack the information needed in the event of a disaster, such as a disastrous earthquake, this could lead to social chaos, including unwanted rumors and outrages, or could disrupt rescue and relief activities ${ }^{1,2}$. In Japan, by law in principle, self-help or mutual assistance is required immediately after a disaster, and local residents are required to make judgments for action on their own. Although disaster information systems are gradually being organized at the municipal level, actual emergency evacuation areas and essential information for local citizens are still not sufficiently ready for provision at this stage. ${ }^{3}$ In this study, we established and evaluated a service infrastructure with an autonomous wireless network, aiming at providing services to collect and deliver disaster information, which will be required by local residents.
\end{abstract}

Keywords: earthquake, disaster victims, distributed autonomous system, wireless network.

\section{Introduction}

In the event of a disaster, such as a disastrous earthquake, information provision is effective in preventing chaos at the scene. Therefore, timely and accurate information collection and delivery services are essential. These services allow prompt rescue and relief activities and appropriate information delivery to local residents. Thus, it is urgent to establish a system to enable these services. The systems proposed so far are ones with Internet or mobile phone connections or with ad-hoc wireless LAN networks ${ }^{4,5}$. We call such systems communication channel dependent systems, which require communication channels or establish communication channels between clients and servers via an ad-hoc network. From the perspective of an information service, such systems that accumulate information in PDAs and send it via an ad-hoc network when a communication channel is established are also regarded as communication channel dependent systems.

There are two issues of concern regarding this system: 1) the system is not available until a communication channel is established, and 2) as users access the server to gain information, the intense access may lower server performance or cause 
communication channel congestion. In this study, we would like to propose an approach to resolve these issues. As it is difficult to generalize the situations of earthquake disasters, we set the following assumptions: (a) assuming a strong earthquake of approximate magnitude 7 in a residential area, (b) all the lifelines including electricity and communication channels stopped functioning, (c) lines for land phones and mobiles are congested and not working, and (d) the proposed system (hereafter called "the system") can be preliminarily placed.

\section{Method}

In order to resolve the above issues, we placed servers, which store information, closed to users in this study. By doing this, the system can run without a communication channel established, and the service can be continuously provided even with a narrow bandwidth. Since disaster information system users, such as local residents, shelter authorities, rescue and relief crews, and municipal employees are diverse and geographically dispersed, multiple servers are required to meet the condition in which servers must be placed close to users. Each server must hold information and be synchronized with each other. Also, they must independently run, communicate with each other, and dynamically detect others in case some are damaged in a disaster.

The service infrastructure consists of many small sub-systems. Each of these systems independently provides information collection and delivery services. Also, these sub-systems can autonomously work together with other sub-systems to exchange information. By appropriately allocating sub-systems within a region, regional information is continuously shared, which can solve the issue of information shortages in a disaster. We adopted general consumer hardware products that are supposed to work approximately 72 hours with batteries. Though each hardware product itself is not robust, they are all independent so even if some of them are damaged, they do not affect others. Also, as it is allowed to dynamically add subsystems, damaged ones can be easily replaced to immediately recover the entire service.

The network configuration of this system is illustrated in the Fig. 1. The system is formed by a group of small servers (hereafter called nodes) with server abilities and dynamic communication functions. Each node can function as a web server and allows clients (e.g. PCs and PDA) to connect to register or browse information. Nodes also detect others within the communication range to synchronize information. With these functions, the system can still work as an integrated unit even when part of the hardware is damaged in a disaster.

Fig. 2 illustrates the hardware configuration of the nodes. We selected only devices that can function approximately 72 hours with either dry-cell or rechargeable batteries. In addition, dedicated PCs can be equipped with server functions for information entries. This means that users can initiate the registration of information even if no communication channels are established. 


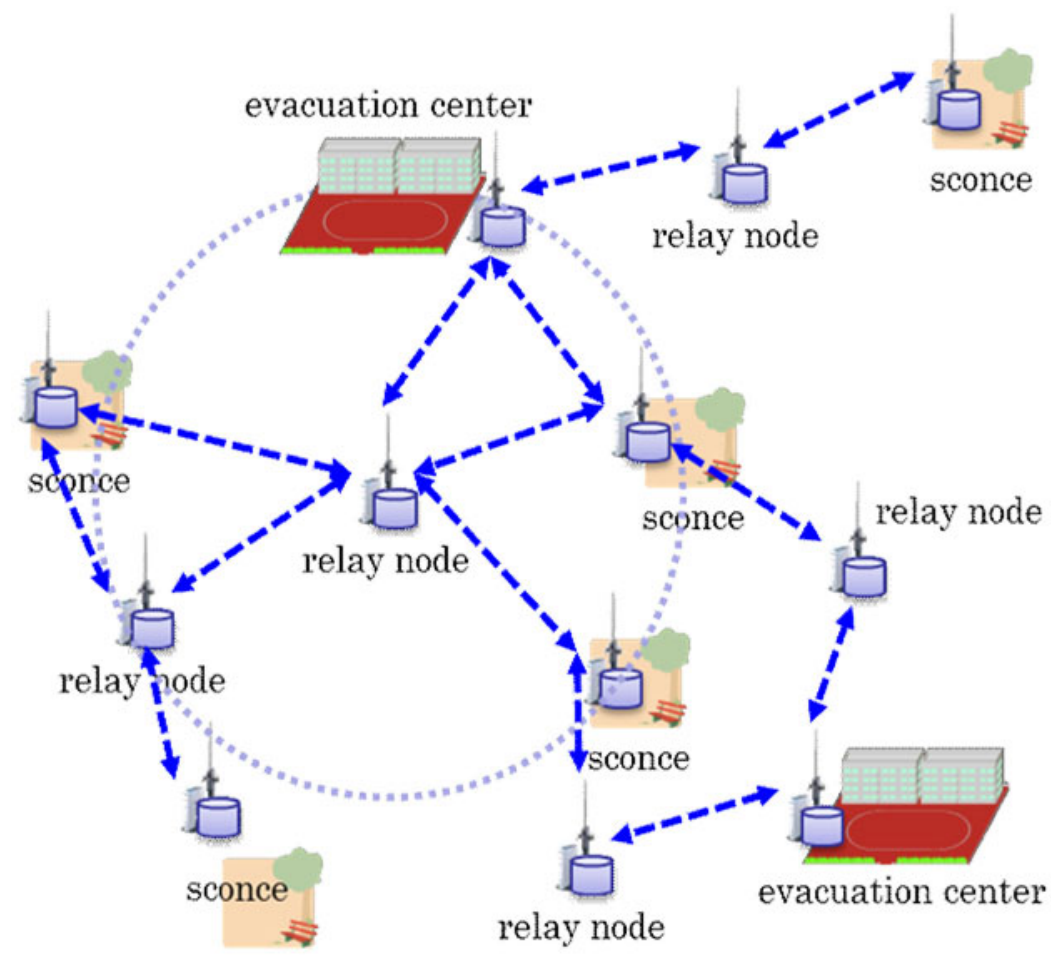

Fig. 1. Whole image of the system: all nodes search another sub system, and then they communicate to each other in order to exchange information they had
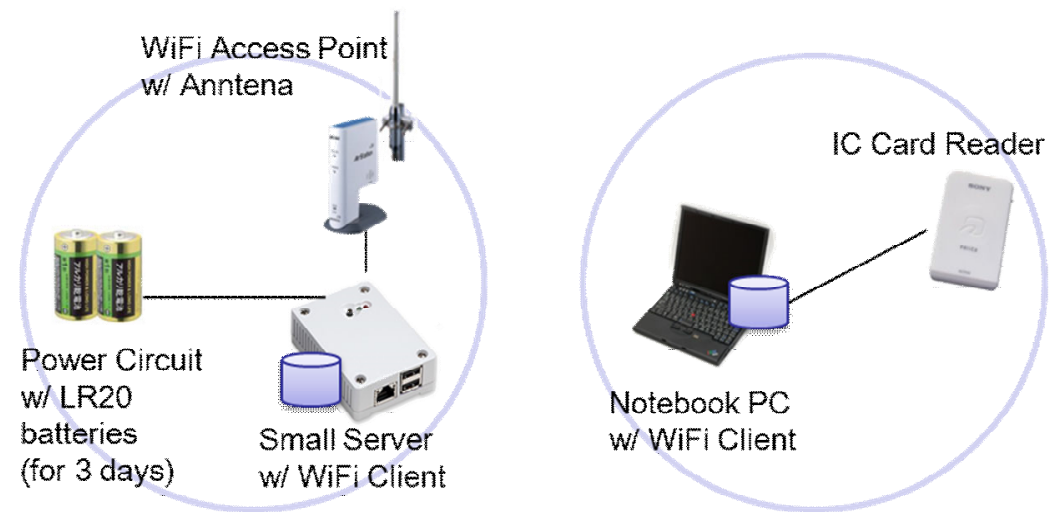

Fig. 2. Elements of the nodes 


\section{Evaluation Experiments}

We conducted a field test and software simulation for the evaluation experiments. In the field test, we developed a prototype and tested operational capabilities by connecting the network between evacuation areas. Software simulation has been performed to check how the information is delivered when the nodes are multiplied to the actual number to be connected.

\subsection{Field Testing}

\section{Apparatus and Materials}

The experiment was conducted at notebook computers and access points with antenna as shown in Fig. 3 These apparatus were located as shown in Fig. 4. The interval to execute process to discovering another nodes and communication was set 60 seconds.

- Node A: IBM ThinkPad X61, Windows Vista Ultimate (32bit), Japanese Edition,

- Node B: IBM ThinkPad X61s, Windows 7 Ultimate (64bit), English Edition,

- Node C: IBM ThinkPad X40, Windows XP Professional (32bit), English Edition,

- Node D: Access Point w/ antenna only.

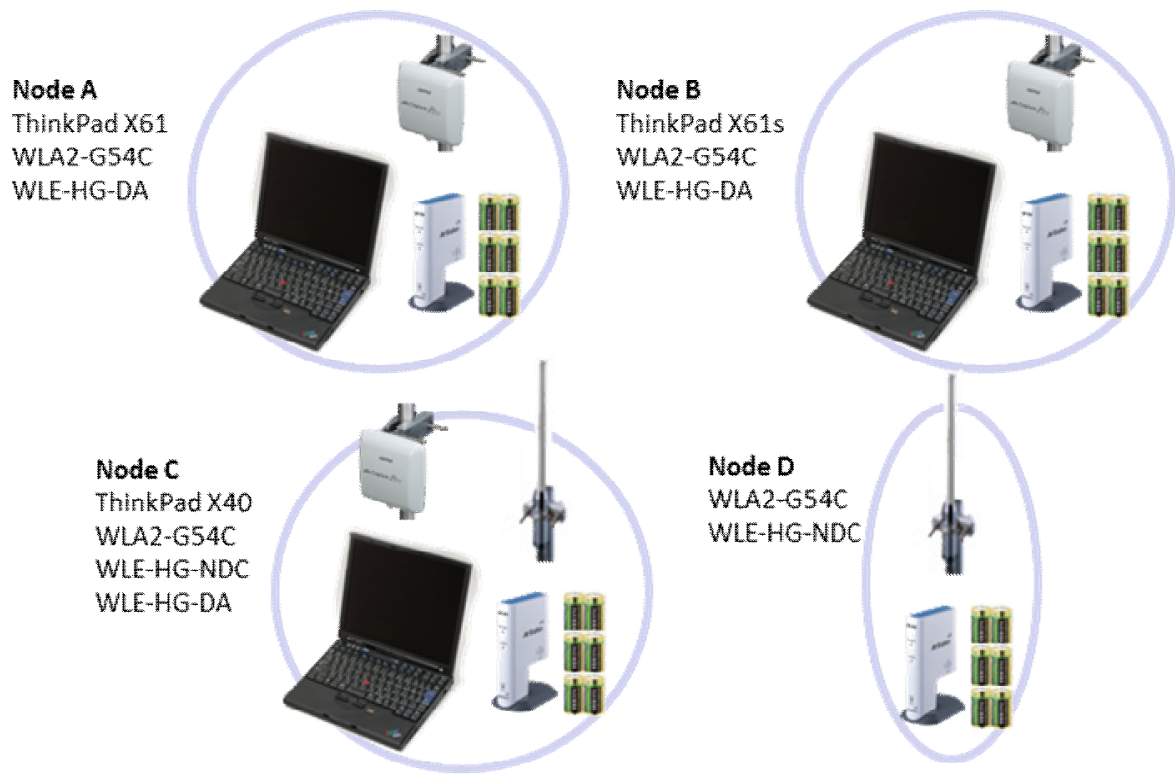

Fig. 3. Apparatus of field testing 


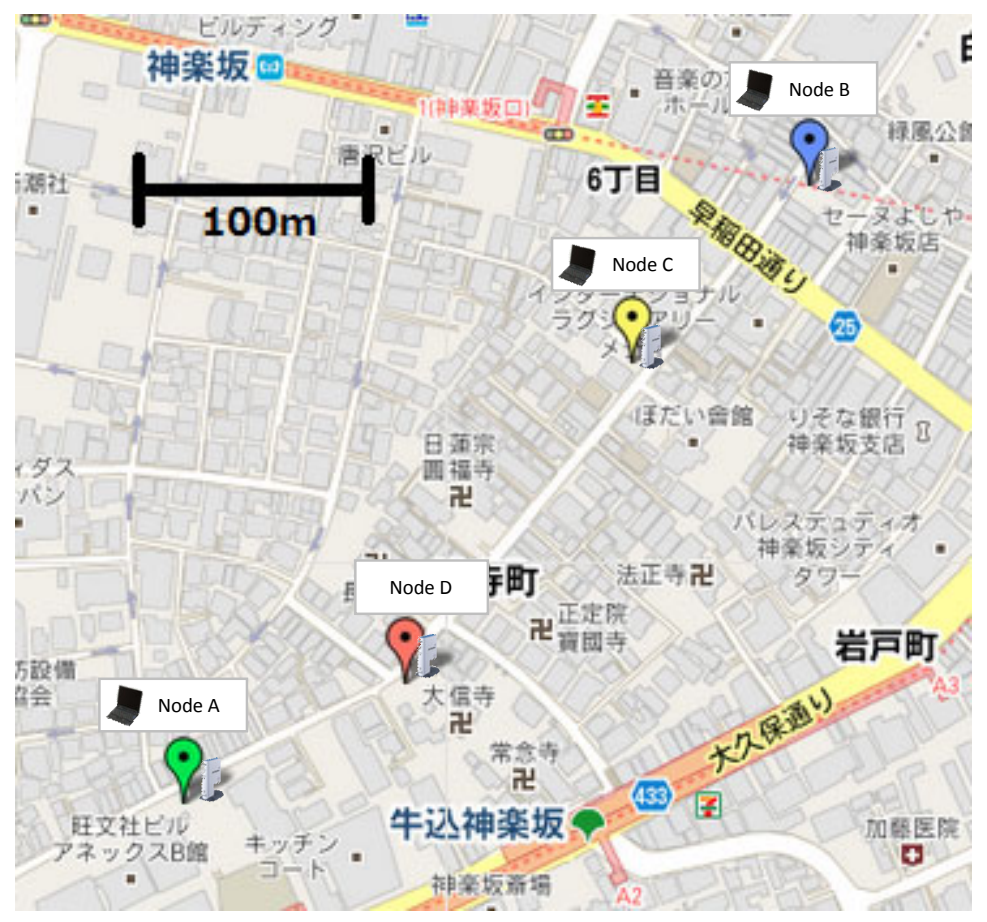

Fig. 4. The layout of the nodes

\section{Procedure}

At first, node A and B were installed as they could not communicate each other. Then, ten data were put into each node. Next, node $\mathrm{C}$ was installed. Then the nodes started communication in order to synchronize information they had. After the synchronization, ten more data were put into each node. The nodes recorded the registered timestamp and the received timestamp for each data as log. These trials were repeated three times.

\subsection{Software Simulation}

\section{Apparatus and Materials}

The experiment was conducted at a notebook computer (IBM ThinkPad X61s, Windows Vista Ultimate (64bit), Japanese Edition, Intel Core2 Duo CPU L7500, 4GBRAM), and the simulator was written in Java language (jdk1.6.0-11). The simulator generated defined node objects, put defined data, and made them 
communicate each other. Each node objects recorded the time when they received all of the data. The parameters (required time to search, connect, and transfer data) were taken from actual measurements.

\section{Procedure}

At first, the node objects and data were defined in text files that contained comma separated values, and then the parameters were defined in properties file of Java language. Next, the simulator was executed ten times.

\section{Result and Discussion}

\subsection{Field Testing}

At first, node A and B accepted user input without any other external network connection. These show the sub-systems have a feature of autonomous controllability. All of the former data were synchronized, after node $\mathrm{C}$ was installed. Node $\mathrm{C}$ was dynamically detected by Node A and B, then they communicated each other. This shows the system has a feature of autonomous coordinability. These two features are important to our system.

Table 1 shows the average of required period to synchronize the latter 10 data. Logically, it takes maximum 240 seconds to synchronize the data. There were 3 nodes in the network, thus, node $\mathrm{C}$ received data within 120 seconds, and then sent them within 120 seconds. It depended on the delay between input timing and invocation timing of synchronization process. The result was nearly half of maximum period. The information synchronize periods were reasonable.

Table 1. The average of required period to transfer 10 data

\begin{tabular}{|l|r|r|}
\hline & 1st 10 data[sec] & next 10 data[sec] \\
\hline Node A->C & 96.3 & 90.7 \\
\hline Node C->B & 102.4 & 95.9 \\
\hline Node A->B & 138.2 & 125.3 \\
\hline
\end{tabular}

\subsection{Software Simulation}

Fig. 5 shows the period required to distribute hundred data from the center node. It indicates the performance is well to far nodes; however, to the nearby nodes of the center node is not satisfied. This caused the communication was serialized; the center node could not communicate to the others, when the number of the nearby nodes was three or more. This point of the system should be improved. Anyway, our target city requires two hundred nodes in order to cover the city, thus, the result indicates the information will be shared in the city within thirty minutes. 


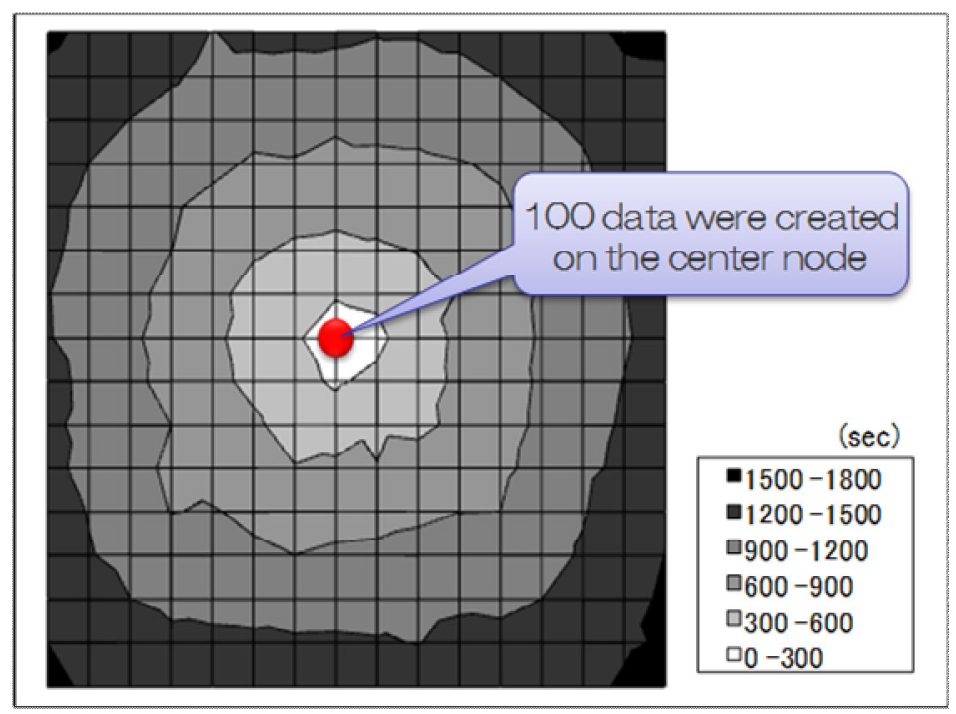

Fig. 5. The period required to distribute hundred data

\section{Conclusion}

We developed information system for disaster victims as the distributed autonomous system using wireless network, and evaluated it. We could confirm the two important feature of our system. First, autonomous controllability increases the availability of collecting information at early time of the disaster. Second, autonomous coordinability helps gradual recovery of whole our system. The data transferring (synchronization) time were reasonable in order to use our system in our target city. Results of this study will be of service to construct disaster information systems for inhabitants.

\subsection{Future Work}

More comprehensive and multifactorial field testing is required. In order to install and use our system actually, more complex geographical layout is required and more nodes are needed. Thus we should evaluate our system in near situation of real.

Investigation of the information needed in a time of disaster is required. The information differs from one that is gathered by autonomous. And more, the user interfaces for the gathering / providing the information should be considered.

\section{References}

1. Osamu, H., et al.: Disaster information and social psychology. Hokuju Shuppan, 177 (2004)

2. Osamu, H.: Hanshin-Awaji (Kobe) Earthquake investigation report in 1995-1. Institute of Socio-Information and Communication Studies, the University of Tokyo (1996) 
3. Sakae, Y.: The Providing Disaster Information Services in Ubiquitous Days. Journal of the Society of Instrument and Control Engineers 47(2), 125-131 (2008)

4. Nobuo, F., et al.: Intercommunications system "AnSHIn-system" and mobile disaster information unit "AnSHIn-Kun. AIJ J. Technol. Des. (12), 227-232 (2001)

5. Yusuke, T., et al.: A wireless mesh network testbed in rural mountain areas. In: The Second ACM International Workshop on Wireless Network Testbeds, Experimental Evaluation and Characterization, pp. 91-92 (2007)

6. The Headquarters for Earthquake Research Promotion, http: / /www. jishin.go.jp

7. Cabinet Office, Government of Japan, http://www. bousai .go.jp 